\title{
Is local consolidative therapy adequate for the treatment of oligometastatic non-small cell lung cancer?
}

\author{
Taichiro Goto \\ Lung Cancer and Respiratory Disease Center, Yamanashi Central Hospital, Yamanashi, Japan \\ Correspondence to: Taichiro Goto, MD, PhD. Lung Cancer and Respiratory Disease Center, Yamanashi Central Hospital, Yamanashi, 400-8506, Japan. \\ Email: taichiro@1997.jukuin.keio.ac.jp. \\ Provenance: This is an invited article commissioned by the Section Editor Xiaozheng Kang (Department of Thoracic Surgery, Beijing Cancer \\ Hospital, Peking University, Beijing, China). \\ Comment on: Gomez DR, Tang C, Zhang J, et al. Local Consolidative Therapy Vs. Maintenance Therapy or Observation for Patients With Oligometastatic \\ Non-Small-Cell Lung Cancer: Long-Term Results of a Multi-Institutional, Phase II, Randomized Study. J Clin Oncol 2019;37:1558-65.
}

Submitted Aug 14, 2019. Accepted for publication Sep 25, 2019.

doi: $10.21037 /$ jtd.2019.09.80

View this article at: http://dx.doi.org/10.21037/jtd.2019.09.80

Lung cancer with distant metastases is regarded as a systemically spread disease and so is conventionally indicated for systemic chemotherapy treatment. Cancer drug treatment has recently developed rapidly for advanced non-small cell lung cancer (NSCLC) and many pharmacotherapies are available as a treatment choice, including cytotoxic chemotherapy, molecular-targeted therapy, and immunotherapy. In spite of these recent advances, the prognosis for metastatic NSCLC remains poor (1-3). Recently, an oligometastatic state in which local treatment of metastatic lesions leads to long-term local control and improved survival has been reported in many cancers (4). In general, conditions with 1-3 or 1-5 metastatic lesions are defined as an oligometastatic cancer $(5,6)$. Based on this concept, many oligometastatic diseases are now treated by local therapies with curative intent worldwide $(7,8)$. Andrews et al. demonstrated in 2004 that adding stereotactic radiosurgery (SRS) to whole-brain radiotherapy boosted median overall survival (OS) from 4.9 to 6.5 months in patients with a single unresected brain metastasis (9). Resection of liver metastases from colorectal cancer was also reported to improve patient survival and is now regarded as a standard surgical procedure (10). However, the cancer status and treatments varied widely in these studies and most of them retrospectively examined the treatment efficacy for oligometastatic disease, and with small sample size (11-14).

Gomez et al. reported a study to determine whether local consolidative therapy (LCT) such as radiotherapy or surgery improved survival in oligometastatic NSCLC (15). The basic principle of treatment against cancer is that local therapy (e.g., surgery or radiotherapy) is applied to locally confined disease, while systemic drug therapy is applied to distant metastatic disease. The novelty of this study is that it prospectively evaluated the significance of the additional application of local therapy to metastatic sites of oligometastatic NSCLC, regardless of the usual basic principles of cancer therapy. In his multicenter, randomized trial, patients were randomized to maintenance therapy or observation (MT/O), or to LCT for all active disease sites. The study demonstrated that, in patients with oligometastatic NSCLC, LCT prolonged PFS and OS compared with MT/O. This topic warrants further consideration in terms of rationale for the local treatment of oligometastasis.

This RCT was designed for oligometastatic NSCLC with $1-3$ metastatic lesions $(15,16)$. All patients were treated by at least four cycles of platinum-based doublet chemotherapy, or alternatively, targeted epidermal growth factor receptor (EGFR) or anaplastic lymphoma kinase (ALK) inhibitors for 3 months before randomization to the MT/O or LCT arm. The study was terminated early from an ethical standpoint, as there was evidently significant improvement in PFS in the LCT group. Among the LCT patients, radiotherapy was the prevalent treatment option: 24 of 25 patients randomized to the LCT arm received 
some form of radiation therapy, while surgeries for brain, lung, lymph node or adrenal metastasis were performed in 6 patients. Furthermore, no life-threatening or fatal adverse effects were reported in either treatment arm $(15,16)$. In this trial, disease progression was observed in 19 of the 25 patients undergoing LCT and 20 of the 24 patients undergoing MT/O. Although no significant differences were observed in the proportions of patients with and without disease progression, OS and PFS were prolonged by LCT. Originally, oligometastasis was a concept developed as a curative treatment target. However, the findings of this trial demonstrated that LCT was beneficial in controlling disease progression even in oligometastatic patients who were not strictly cured by LCT. Survival period after disease progression significantly differed between the patients originally assigned to receive LCT and those originally assigned to receive MT/O. This observation suggests that LCT may delay the overall progression of NSCLC and influence OS. On the basis of these results, the investigators proposed that LCT should be the standard treatment option for oligometastatic NSCLC. It was a novel, logical, and reasonable proposal, and we considered that this study indeed provided valuable information. However, there are certain limitations in their study design that need to be pointed out. In particular, the trial comprised only 49 patients in total due to early termination, a number which essentially precluded further subgroup analyses.

Nowadays, the number of patients diagnosed with oligometastatic disease is steadily increasing owing to the development of sensitive imaging technologies and effective therapies which have improved the survival of patients diagnosed with cancer $(17,18)$. In fact, $50 \%$ of patients newly diagnosed with stage IV NSCLC are reported to have 1-3 metastases (19). In cases of postoperative recurrence of NSCLC, $33 \%$ and $19 \%$ of patients are reported to have isolated and 2-3 metastases, respectively (19). Thus, remarkably, it is assumed that numerous patients meet the basic definition of oligometastatic NSCLC in actual clinical practice.

Conventionally, oligometastases have been treated by surgical resection, because surgical resection is anecdotally believed to yield survival benefits $(7,8)$. However, recently, SRS and stereotactic ablative body radiotherapy (SABR) have often replaced surgery for the treatment of oligometastasis $(20,21)$. At first, SRS was proved effective for solitary brain metastasis (9), then SABR became a prevalent modality for treating extracranial metastases, partly due to its minimum invasiveness and strong efficacy. As a whole, the use of SABR in patients with extracranial oligometastasis is an emerging and evolving therapeutic approach. In the trial conducted by Gomez et al., the LCT group comprised both patients undergoing SABR (SRS) (56\%) and patients undergoing surgery (24\%). The selection of surgery or SABR may be affected by the organs in which metastasis has occurred, the number of metastases, and/or physical condition of the patients undergoing treatment. Moreover, at present there is no randomized evidence comparing SABR and surgery. Therefore, a randomized clinical trial comparing SABR and surgery in a specific oligometastatic condition is requisite for the establishment of the proper therapeutic choice in the oligometastatic condition.

Meanwhile, Gomez et al. were unable to perform a subgroup analysis due to the small sample size of their trial. Hence, they did not investigate which pathological conditions (e.g., the primary lesion, organs in which metastasis occurred, number of metastases, or timing of metastasis) of the patients with oligometastatic NSCLC best exhibited the benefit of LCT. Previous studies have vigorously searched for clinical prognostic factors for longterm survival after local therapy. Of note, metachronous metastases, a long disease-free interval, a smaller number of metastases and small size have frequently been identified as prognostic factors (22). However, patient selection using only these clinical factors is not accurate, definitive or beneficial in all oligometastatic conditions. Novel biomarkers for predicting treatment response should be urgently established in order to apply this new treatment strategy in the clinical setting. Furthermore, Gomez et al. did not include immunocheckpoint blockade therapy as front-line systemic therapy since their trial commenced in 2012. In the past 5 years, the use of immunotherapy has revolutionized the treatment of NSCLC. Therefore, future studies may examine a combination of immunotherapy and LCT (23). In this context, a large-scale, prospective, and randomized study is warranted to validate the present findings and apply them to clinical practice for the treatment of NSCLC.

Several clinical trials are ongoing to evaluate the use of LCT for oligometastases $(24,25)$. The Stereotactic Ablative Radiotherapy for Oligometastatic Non-Small Cell Lung Cancer (SARON) trial is investigating the impact of adding SABR/SRS to standard chemotherapy for oligometastatic NSCLC (NCT02417662). NRG-LU002 is a multicenter randomized phase II/III trial evaluating the impact of adding SBRT to maintenance systemic therapy versus maintenance systemic therapy alone on the survival of 
patients with oligometastatic NSCLC (NCT03137771). These clinical trials are expected to establish a collective data set for the use of LCT in oligometastatic NSCLC.

Considering the clinical evidence supporting the concept of oligometastasis, we call into account the fact that oligometastasis is an actual therapeutic target rather than a hypothetical disease concept. It is becoming apparent that patients with oligometastatic NSCLC may possibly benefit from LCT. This is a major step forward that overturns the conventional knowledge regarding the treatment of lung cancer. Further clinical trials will better elucidate the current enigmatic efficacy of LCT and guide the treatment of oligometastatic conditions.

\section{Acknowledgments}

The author greatly appreciates Sotaro Otake and Rumi Higuchi for their helpful scientific discussions.

\section{Footnote}

Conflicts of Interest: The author has no conflicts of interest to declare.

Ethical Statement: The author is accountable for all aspects of the work in ensuring that questions related to the accuracy or integrity of any part of the work are appropriately investigated and resolved.

\section{References}

1. Iijima Y, Hirotsu Y, Amemiya K, et al. Very early response of circulating tumour-derived DNA in plasma predicts efficacy of nivolumab treatment in patients with non-small cell lung cancer. Eur J Cancer 2017;86:349-57.

2. Łasińska I, Kolenda T, Teresiak A, et al. Immunotherapy in Patients with Recurrent and Metastatic Squamous Cell Carcinoma of the Head and Neck. Anticancer Agents Med Chem 2019;19:290-303.

3. Sia DIT, Thaung C, O'Hanlon-Brown C, et al. Immune privilege: failure of immunotherapy in controlling metastatic cutaneous melanoma to the eye. Melanoma Res 2018;28:359-62.

4. Hellman S, Weichselbaum RR. Oligometastases. J Clin Oncol 1995;13:8-10.

5. Milano MT, Katz AW, Zhang H, et al. Oligometastases treated with stereotactic body radiotherapy: long-term follow-up of prospective study. Int J Radiat Oncol Biol
Phys 2012;83:878-86.

6. Palma DA, Salama JK, Lo SS, et al. The oligometastatic state - separating truth from wishful thinking. Nat Rev Clin Oncol. 2014;11:549-57.

7. Goto T, Maeshima A, Oyamada Y, et al. Solitary pulmonary metastasis from prostate sarcomatoid cancer. World J Surg Oncol 2010;8:101.

8. Shikata D, Nakagomi T, Higuchi R, et al. Lymph node cancer of the mediastinum with a putative necrotic primary lesion in the lung: a case report. World J Surg Oncol 2018;16:73.

9. Andrews DW, Scott CB, Sperduto PW, et al. Whole brain radiation therapy with or without stereotactic radiosurgery boost for patients with one to three brain metastases: phase III results of the RTOG 9508 randomised trial. Lancet 2004;363:1665-72.

10. Fong Y, Fortner J, Sun RL, et al. Clinical score for predicting recurrence after hepatic resection for metastatic colorectal cancer: analysis of 1001 consecutive cases. Ann Surg 1999;230:309-18; discussion 318-21.

11. De Ruysscher D, Wanders R, van Baardwijk A, et al. Radical treatment of non-small-cell lung cancer patients with synchronous oligometastases: long-term results of a prospective phase II trial (Nct01282450). J Thorac Oncol 2012;7:1547-55.

12. Pasqualetti F, Panichi M, Sainato A, et al. [(18)F]Choline PET/CT and stereotactic body radiotherapy on treatment decision making of oligometastatic prostate cancer patients: preliminary results. Radiat Oncol 2016;11:9.

13. Collen C, Christian N, Schallier D, et al. Phase II study of stereotactic body radiotherapy to primary tumor and metastatic locations in oligometastatic nonsmall-cell lung cancer patients. Ann Oncol 2014;25:1954-9.

14. Iyengar P, Kavanagh BD, Wardak Z, et al. Phase II trial of stereotactic body radiation therapy combined with erlotinib for patients with limited but progressive metastatic non-small-cell lung cancer. J Clin Oncol 2014;32:3824-30.

15. Gomez DR, Tang C, Zhang J, et al. Local Consolidative Therapy Vs. Maintenance Therapy or Observation for Patients With Oligometastatic Non-Small-Cell Lung Cancer: Long-Term Results of a Multi-Institutional, Phase II, Randomized Study. J Clin Oncol 2019;37:1558-65.

16. Gomez DR, Blumenschein GR, Jr., Lee JJ, et al. Local consolidative therapy versus maintenance therapy or observation for patients with oligometastatic non-smallcell lung cancer without progression after first-line systemic therapy: a multicentre, randomised, controlled, 
phase 2 study. Lancet Oncol 2016;17:1672-82.

17. Mehta N, Mauer AM, Hellman S, et al. Analysis of further disease progression in metastatic non-small cell lung cancer: implications for locoregional treatment. Int J Oncol 2004;25:1677-83.

18. Yao HH, Hong M, Corcoran NM, et al. Advances in local and ablative treatment of oligometastasis in prostate cancer. Asia Pac J Clin Oncol 2014;10:308-21.

19. Singh D, Yi WS, Brasacchio RA, et al. Is there a favorable subset of patients with prostate cancer who develop oligometastases? Int J Radiat Oncol Biol Phys 2004;58:3-10.

20. Palma DA, Louie AV, Rodrigues GB. New Strategies in Stereotactic Radiotherapy for Oligometastases. Clin Cancer Res 2015;21:5198-204.

21. Renaud S, Falcoz PE, Olland A, et al. Is radiofrequency ablation or stereotactic ablative radiotherapy the best

Cite this article as: Goto T. Is local consolidative therapy adequate for the treatment of oligometastatic non-small cell lung cancer? J Thorac Dis 2019;11(10):E154-E157. doi: 10.21037/ jtd.2019.09.80 treatment for radically treatable primary lung cancer unfit for surgery? Interact Cardiovasc Thorac Surg 2013;16:68-73.

22. Spelt L, Andersson B, Nilsson J, et al. Prognostic models for outcome following liver resection for colorectal cancer metastases: A systematic review. Eur J Surg Oncol 2012;38:16-24.

23. Goto T. Radiation as an In Situ Auto-Vaccination: Current Perspectives and Challenges. Vaccines (Basel) 2019. doi: 10.3390/vaccines7030100.

24. Otake S, Goto T. Stereotactic Radiotherapy for Oligometastasis. Cancers (Basel) 2019. doi: 10.3390/ cancers11020133.

25. Palma DA, Haasbeek CJ, Rodrigues GB, et al. Stereotactic ablative radiotherapy for comprehensive treatment of oligometastatic tumors (SABR-COMET): study protocol for a randomized phase II trial. BMC Cancer 2012;12:305. 